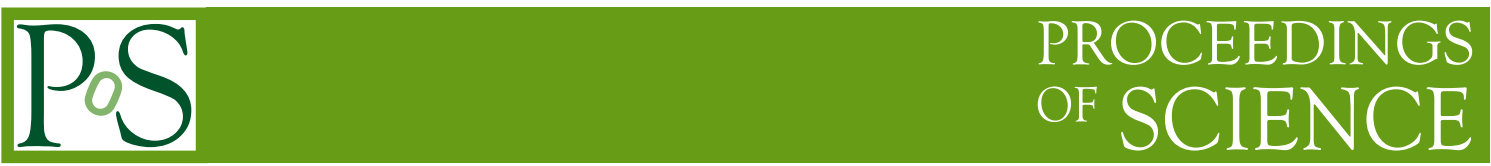

\title{
Pamela main results after 5 years of data taking
}

\author{
O. Adriani, on behalf of the PAMELA Collaboration* \\ Università degli Studi di Firenze and INFN Sezione di Firenze \\ E-mail: adriani@fi.infn.it
}

The PAMELA satellite-borne experiment was launched from the Bajkonur cosmodrome in June 2006. The combination of a permanent magnet silicon strip spectrometer and a silicon-tungsten imaging calorimeter allows precision studies of the charged cosmic rays to be conducted over a wide energy range with high statistics. After five years of successfully operation in space, the data gathered by the PAMELA experiment are showing very interesting features in cosmic rays spectra. This paper shortly present the latest measured fluxes of particles (protons, heliums and electrons) and antiparticles (positrons), that may challenge the current paradigm of cosmic-ray acceleration and propagation in the Galaxy.

The 2011 Europhysics Conference on High Energy Physics, EPS-HEP 2011,

July 21-27, 2011

Grenoble, Rhône-Alpes, France

${ }^{*}$ Speaker. 


\section{The PAMELA experiment}

The PAMELA detector has been launched in space by a Soyuz-U rocket on June 15th 2006 from the Baikonur cosmodrome in Kazakhstan, and is continously taking data since July 2007. The satellite orbit has an altitude varying between $350 \mathrm{~km}$ and $600 \mathrm{~km}$, at an inclination of $70^{\circ}$. The experiment has been designed to precisely measure the spectra of primary and secondary components of the cosmic radiation in a wide energy range, with a particular focus on antiparticles (antiprotons and positrons). A detailed description of the PAMELA experiment and of it's expected physics performances can be found in [1]. Here we will simply give a short description of the apparatus, with main emphasis on the sub-detectors relevant for the physics analysis described in the following sections.

The detector is approximately $1.3 \mathrm{~m}$ high, has a mass of $470 \mathrm{~kg}$ and an average power consumption of $355 \mathrm{~W}$. The central part of the apparatus is a magnetic spectrometer consisting of a permanent magnet and a silicon tracking-system, composed by six equidistant planes of doublesided microstrip silicon detectors. The dimensions of the permanent magnet define the geometrical factor of the PAMELA experiment to be $21.5 \mathrm{~cm}^{2} \mathrm{sr}$. The magnetic spectrometer is used to measure the rigidity $(\mathrm{R}=\mathrm{cp} / \mathrm{Ze}$ ) of particles, up to to a maximum detectible rigidity (MDR) exceeding 1 TV. In flight, the deflection measurement of the tracking system is cross-checked with the energy measurement of the calorimeter for high- energy electrons and positrons.

The Time of Flight (ToF) system comprises six layers of fast plastic scintillators arranged in three double planes, and is used to determine particle velocities. The measured time-of-flight resolution (better than $300 \mathrm{ps}$ ) allows $\mathrm{e}^{-}\left(\mathrm{e}^{+}\right)$to be separated from $\bar{p}$ (p) up to $1 \mathrm{GeV} / \mathrm{c}$. Albedo particles can also be rejected with a significance of 60 standard deviations.

The sampling electromagnetic calorimeter comprises 44 single-sided silicon planes interleaved with 22 plates of tungsten absorber. The total depth of the calorimeter is $16.3 \mathrm{X}_{0}(0.6 \lambda)$. The longitudinal and transverse segmentation of the calorimeter allows a high rejection power for electromagnetic showers against interacting and non-interacting hadrons.

The PAMELA experiment is completed by two anticoincidence systems, by a shower tail catcher scintillator and by a neutron detector, to help the calorimeter in the selection of the particles.

\section{2. $p$ and He absolute fluxes}

Protons and helium nuclei are the most abundant components of the cosmic rays; precise measurements of their fluxes are important to better understand the acceleration and propagation mechanism of cosmic rays in the Galaxy and to constrain the propagation models, that are essential for Dark Matter searches. These measurements represent a big challenge from the experimental point of view, since a 1-2\% precision in the absolute fluxes is necessary, implying a detailed study and understanding of the detector systematics. Moreover, the necessity to cover a very broad energy range (from $100 \mathrm{MeV}$ up to more than $1 \mathrm{TeV}$ and the long term exposure require particular care in the analysis procedure. The results reported here have been published in [2], and are based on the high statistic sample of data gathered by PAMELA between 2006 and 2008.

Figure 1 shows the absolute proton and helium fluxes (multiplied by $\mathrm{E}^{2.7}$ ) as function of the kinetic energy measured by PAMELA above $1 \mathrm{GeV} / \mathrm{n}$, compared with some of the previous mea- 
surements (see [2] for the references to these measurements). PAMELA data cover the energy range $1 \mathrm{GeV}-1.2 \mathrm{TeV}(1-600 \mathrm{GeV} / \mathrm{n}$ for $\mathrm{He})$. The fluxes are expressed in terms of kinetic energy per nucleon. Error bars are statistical only, the shaded area represents the estimated systematic uncertainty. From these data we can clearly observe a change of spectral slope in the high energy region, both in the proton and the helium spectra. This feature is more evident from Figure 2, where the same data are plotted as function of the rigidity, in the range $10 \mathrm{GV}-1.2 \mathrm{TV}$. The gray shaded area represents the estimated systematic uncertainty and the pink shaded area represents the contribution due to tracker alignment. The straight (green) lines represent fits with a single power law in the rigidity range 30-240 GV. The red curves represent the fit with a rigidity dependent power law (30-240 GV) and with a single power law above $240 \mathrm{GV}$. An abrupt change of the spectral slope is present in both spectra for a rigidity of $\simeq 230-240 \mathrm{GV}$; standard propagation models widely used in cosmic ray physics do not show such behavior, implying that a refinement of the models is certainly necessary to precisely describe the propagation phenomena in the galaxy.

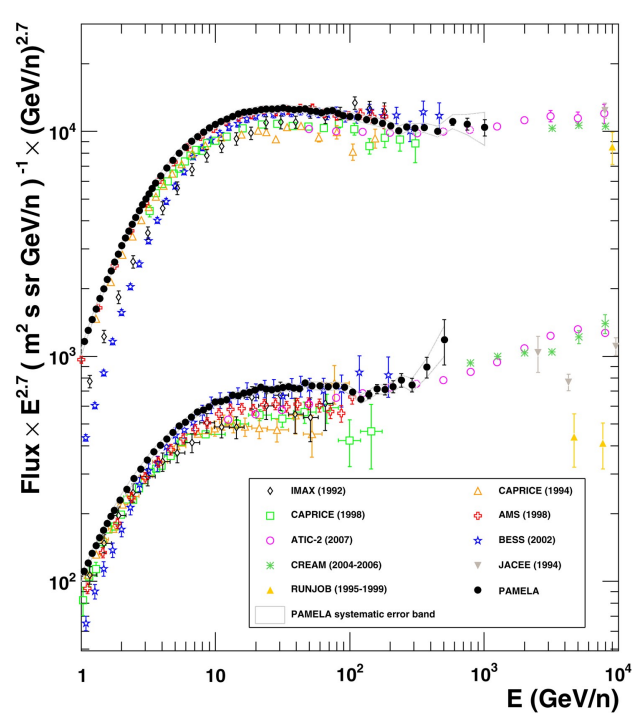

Figure 1: Proton and helium spectra as function of the kinetic energy measured by PAMELA, compared with some of the previous measurements (from [2]).
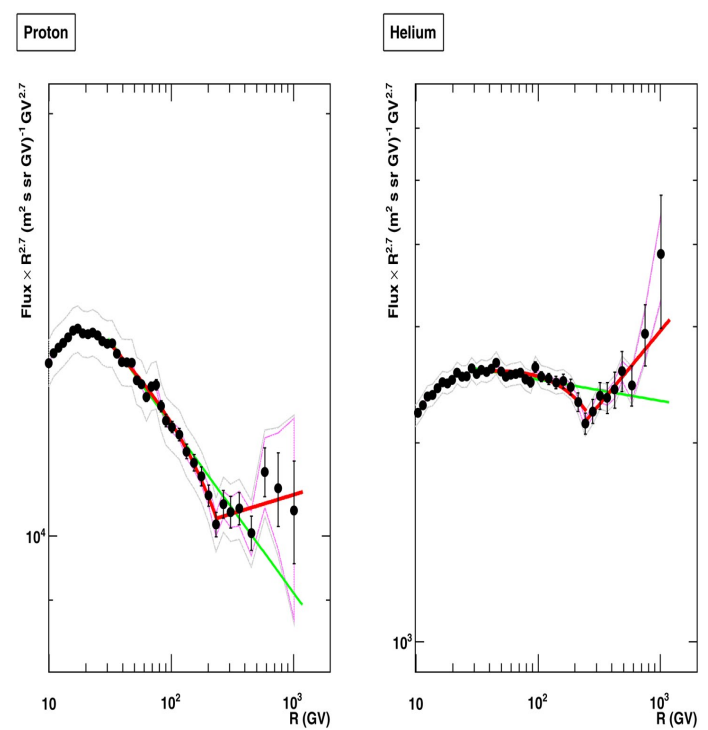

Figure 2: Proton and helium spectra as function of the rigidity. For a detailed description of the fitting lines, see text (from [2]).

\section{Electrons and positrons absolute fluxes}

Precision measurements of the electron component in the cosmic radiation provide additional important information about the origin and propagation of cosmic rays in the Galaxy. The PAMELA experiment is particularly well suited for this type of measurement, due to the excellent performances of the calorimeter in the electromagnetic particles selection in the huge hadron background. Additionally, the momentum precisely measured by the magnetic spectrometer allows a very clean separation between electrons and positrons up to high energies, making PAMELA the most important experiment for the separate measurement of electrons and positrons fluxes. The 
latest results are reported in Figure 3. The electron absolute flux (upper red points, from [3]) has been measured between $1 \mathrm{GeV}$ and $625 \mathrm{GeV}$, and it can be fairly well described with a single power law energy dependence with spectral index $-3.18 \pm 0.05$ above the energy region influenced by the solar modulation (> $30 \mathrm{GeV}$ ). The positron absolute flux up to $100 \mathrm{GeV}$ (lower red squares) has been extracted from the published positron fraction [4] and from the electron measured flux. Our

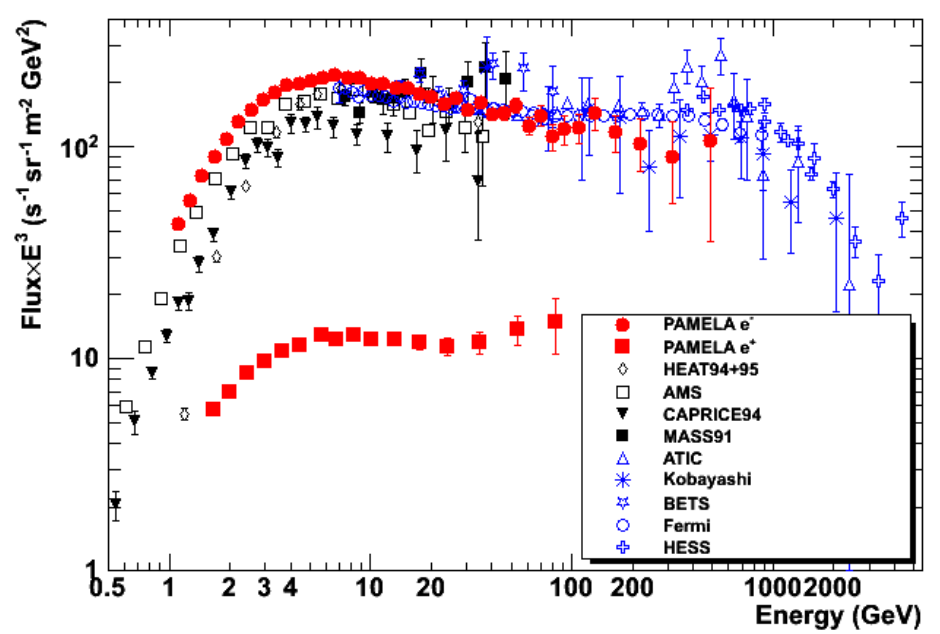

Figure 3: Electrons (upper red points) and positrons (lower red squares) absolute fluxes measured by PAMELA. The electrons data are from [3], while the positron flux has been extracted from the published positron fraction [4] and from the electron flux. The electron flux is compared with some other recent measurements of the $\mathrm{e}^{-}$or $\left(\mathrm{e}^{-}+\mathrm{e}^{+}\right)$fluxes (see [3] for the references to the measurements).

results are not inconsistent with the standard model of cosmic ray acceleration and propagation in the Galaxy, since no significant spectral features are observed in the electron flux, and the data can be interpreted in terms of conventional diffusive propagation models. However, as described in [3], there is some tension between the data and the GALPROP [5] prediction that points to possible refinements of the propagation models and might require additional sources of cosmic rays in the galaxy. If we reasonably assume that these hypothetical additional sources can contribute in equal amount to the electron and positron component, we could easily explain the anomalous positron fraction that has been measured by PAMELA [4], and that is responsible of the evident slope change in the positron flux reported in Figure 3.

\section{References}

[1] P. Picozza et al., Astropart. Phys. 27 (2007) 296

[2] O. Adriani et al., Science - 332 (2011) 6025

[3] O. Adriani et al., Phys. Rev. Lett. 106, 201101 (2011)

[4] O. Adriani et al., Astropart. Phys. 34 (2010) 1

[5] A. W. Strong and I. V. Moskalenko, Astrophys. J. 509, 212 (1998) 